\title{
La compasión: clave en la renovación de la atención en salud
}

\author{
Compassion: key in the renewal of health care
}

\author{
Paula Bedregal ${ }^{1,2}$, Victoria Lermanda' ${ }^{1}$, Gonzalo Brito-Pons ${ }^{3}$
}

\section{Resumen}

Introducción: durante el último tiempo, las prácticas de atención en salud han experimentado un profundo cambio en la medida que los avances científicos y tecnológicos han alcanzado y modificado la clásica relación médico-paciente. El panorama moderno y las presiones e influencias económicas sobre el ejercicio profesional han conducido, además, a una manifiesta insatisfacción respecto al servicio, tanto de usuarios como de profesionales. Ante esta situación, la compasión podría constituirse en un recurso clave para redirigir nuevamente el foco de la atención hacia la persona. Material y método: se realiza una revisión bibliográfica con el propósito de ilustrar sobre esta capacidad, así como invitar a la reflexión sobre la misma. Resultados: el desarrollo de la compasión permite mejorar el trato con los pacientes al mismo tiempo que contribuye al autocuidado de los profesionales de la salud. La evidencia científica disponible al respecto ha permitido comprender la naturaleza neurobiológica de esta capacidad, así como los múltiples beneficios de su desarrollo e integración a la práctica clínica. Conclusión: existe una necesidad urgente de llevar a cabo una transformación de la atención de salud, la cual no debe plantearse de manera exclusiva a partir de los requerimientos estructurales-materialistas del sistema, sino que también debe reivindicar la importancia de implementar cambios personales y organizacionales que se direccionen hacia una humanización del servicio, para centrarlo efectivamente en la persona.

Palabras clave: compasión; educación médica; práctica clínica; salud pública.

\begin{abstract}
Introduction: In recent times, health care has undergone a deep change in the way scientific and technological advances have reached and modified the traditional doctor-patient relationship. The modern context and the economic pressures influence how medical care is performed and have also led to dissatisfaction, both from users and professionals, with health care services. In response to these, compassion could be a key resource to redirect the attention focus back to the whole person. Material and methods: A bibliographic review was carried with the purpose to illustrate this compassionate motivation, as well as to invite to reflect about it. Results: Developing compassion in healthcare professionals and institutions improve the quality of health care and, at the same time, contributes to the professional's self-care. Available scientific evidence has allowed us to understand the neurobiological basis of compassion, as well as the multiple benefits of its development and integration into clinical practice. Conclusion: It is both necessary and urgent to carry out a transformation of the health care system in the direction of compassionate care, which must not be formulated exclusively from the structural-materialistic requirements of the system, but also must to claim the importance of implementing personal and organizational changes oriented towards humanization of service.
\end{abstract}

Keywords: compassion; medical education; clinical practice; public health.

Fecha de envío: 25 de junio de 2019 - Fecha de aceptación: 23 de marzo de 2020

La atención en salud se encuentra actualmente en una situación de cambio y reformulación profunda. Los avances médicos, la inclusión de tecnologías basadas en inteligencia artificial como la medicina de precisión (Juengst et al., 2016), están cambiando nuestras prácticas y modificando en parte la tradicional relación médico-paciente (Hill, 2009). Asociado a este fenómeno nos encontramos con que las consideraciones económicas en el ejercicio profesional, en particular las crisis económicas de países

(1) Departamento de Salud Pública. Escuela de Medicina. Pontificia Universidad Católica de Chile.

(2) Unidad de Medicina y Salud Integrativa. Escuela de Medicina. Pontificia Universidad Católica de Chile.

(3) Centro Cultivar la Mente. España.

Autor de correspondencia: pbedrega@med.puc.cl 
desarrollados, impusieron una práctica basada en la eficiencia que ha generado insatisfacción tanto por parte de usuarios como de profesionales (Mladovsky et al., 2012).

Por otro lado, la demanda que suscita la alta y creciente prevalencia de enfermedades crónicas y problemas en el ámbito de la salud mental, sumado al deseo de los usuarios de contar con una mejor salud y calidad de vida, han incrementado la tensión del sistema (Thompson et al., 2014). También se hace cada vez más clara la necesidad de implementar intervenciones menos agresivas y con menos efectos adversos para garantizar la seguridad a los pacientes y contribuir a la reducción de gastos innecesarios (Kuhn et al., 2016).

Estos factores contextuales permiten explicar el hecho de que más de un tercio de la población chilena recurra al uso de medicinas alternativas y terapias complementarias (Bedregal et al., 2016). Los usuarios valoran particularmente el espacio de acogida y el trato brindados hacia su persona en este contexto. La discusión internacional sobre este fenómeno ha puesto de manifiesto las carencias de un sistema médico que ha centrado su interés y atención en la enfermedad más que en la persona que encarna y experimenta dicha condición. Esto pese a que la definición de calidad de la atención destaca la relevancia de centrarse en el paciente (Constand et al., 2014) (tabla 1).

Tabla 1: Criterios de calidad de la atención del instituto de medicina norteamericano.

\begin{tabular}{l}
\hline \multicolumn{1}{c}{ Criterios IOM } \\
\hline Efectividad (resultados terapéuticos positivos) \\
\hline Seguridad (control de efectos adversos) \\
\hline Oportunidad (atención otorgada cuando se necesita \\
\hline Eficiencia (atención otorgada con buen uso de recursos) \\
\hline Equidad en el acceso (que no genere asimetría en \\
acceso a servicios de salud) \\
\hline Centrado en el paciente (según sus necesidades) \\
\hline
\end{tabular}

Fuente: Institute of Medicine (2011)

\section{La compasión en el contexto de renovación del quehacer en salud}

Ante el desafío que implica lograr implementar servicios que operen centrados en la persona, la respuesta y solución podría ser la compasión. El conocimiento científico disponible al respecto nos muestra preliminarmente los beneficios que conllevan su desarrollo y su relevancia específica en el ámbito de la salud.

En primer lugar, cabe señalar que la definición de lo que es compasión y lo que se entiende por el concepto no ha estado exento de debate. La Real Academia de la Lengua Española (RAE) ofrece dos definiciones: en primera instancia la precisa como "el sentimiento de conmiseración y lástima que se tiene hacia quienes sufren penalidades o desgracia"; es decir, la compasión es comprendida como un sentimiento de lástima. Este concepto no parece interpelarnos para efectos del trabajo en salud y, así definida, no es el objeto de estudio en el ámbito de la psicología y la medicina. Sin embargo, la RAE ofrece una segunda acepción: "el sentimiento de tristeza o malestar que produce el ver padecer a alguien y que impulsa a aliviar, remediar o evitar su dolor o sufrimiento". Es esta definición la que más se acerca al entendimiento que actualmente se sostiene desde la psicología de la compasión, y que es la que se está usando en ciencia: la compasión es la sensibilidad y apertura hacia el sufrimiento propio y de los demás, unido a la motivación de aliviarlo y prevenirlo (Gilbert, 2015; Jinpa, 2016; Ricard, 2016). En esa línea, es interesante notar cómo, desde la psicología, la relevancia no está puesta en el acto de sentir tristeza o malestar, sino más bien en la apertura sensible hacia el otro y la motivación de contribuir al alivio de su sufrimiento. Es precisamente la sensibilidad y la motivación positiva lo que hace que la compasión sea un recurso importante no solo en el cuidado de los pacientes, sino también en el autocuidado de los profesionales de la salud (Brito-Pons \& Librada-Flores, 2019).

La compasión actualmente se entiende como una capacidad humana que posee varios atributos que la hacen única (Hangartner, 2015); integrando procesos afectivos, cognitivos y conductuales (Figura 1), como son:

1. Ser capaz de sentir lo que siente el otro: proceso afectivo.

2. Comprender lo que le ocurre al otro: proceso cognitivo.

3. Desear profundamente aliviar el sufrimiento del otro: proceso volitivo-actitudinal

4. Actuar en concordancia con lo anterior: proceso conductual.

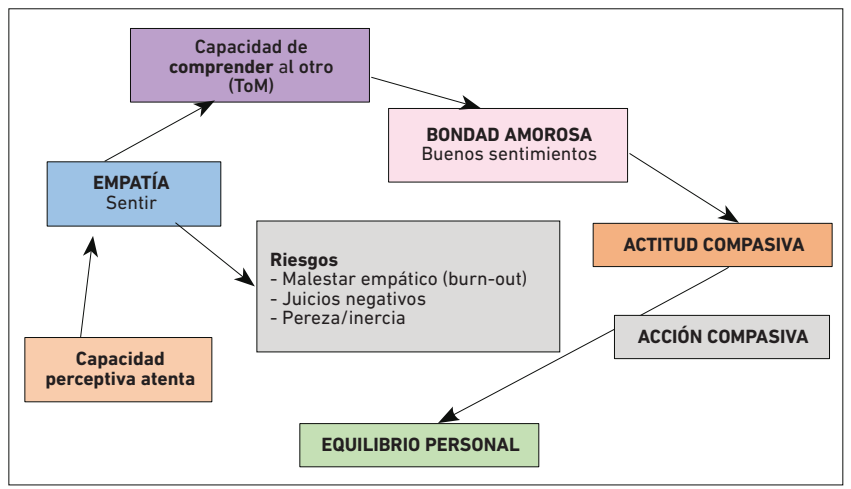

Figura 1: La compasión es una capacidad de auto-regulación emocional que favorece conductas altruistas. 
Para actuar compasivamente, sin duda se requiere coraje y fortaleza interior; el que compadece se enfrenta a sus miedos en una apertura genuina y personal hacia el otro. La lástima, entendida a partir de la primera definición otorgada por la RAE, nos coloca en una posición de superioridad respecto del otro; no asume que todos compartimos una misma humanidad. La lástima además también puede constituirse como una respuesta de distanciamiento defensivo frente al miedo que puede provocar entrar en contacto con el sufrimiento de otro.

Es necesario distinguir claramente la compasión de otros conceptos como son: la teoría de la mente (proceso cognitivo que permite inferir pensamientos, creencias e intenciones de otros); la apreciación (proceso cognitivo de valoración del otro); la empatía (proceso de comprender y sentir lo que siente el otro); el contagio emocional (proceso afectivo de sentir lo que siente el otro perdiendo los límites personales) y la lástima (sentimiento de malestar por un otro a quién se considera inferior desde una posición de superioridad personal).

Existen tres direcciones en las cuales se puede experimentar la compasión: compasión hacia uno mismo (auto-compasión), compasión hacia otros y recibir compasión de los otros (Gilbert et al., 2017). También podemos incluir una cuarta dirección, complementaria a las que propone Gilbert, y es desde y hacia la comunidad (la compasión justa o el actuar justo) (Gomez-Batiste et al., 2018).

La compasión es un proceso dinámico, puesto que las diversas direcciones o flujos de experiencia compasiva interactúan entre sí y favorecen o limitan (si no hay desarrollo) los otros flujos. Un aspecto importante a tener en cuenta, por ejemplo, es la relevancia que tiene el aprender a recibir la compasión de otros, puesto que nos enseña vivencialmente lo que es ser acogido compasivamente. Por otra parte, la autocompasión, que es la capacidad de responder con calidez frente a nosotros mismos en momentos de sufrimiento, aceptándonos, siendo empáticos con nuestra existencia y teniendo la intención de apoyarnos con cariño, es clave para poder entregar compasión de manera sostenible y sin agotarnos. Para Neff \& Germer (2017), la autocompasión implica no tenernos lástima, no quedarnos atrapados en nuestro relato mental; no ser autoindulgente, no escabullirnos de la realidad. La autocompasión no es tampoco auto-exigencia ni tener alta autoestima, comprendiendo esta última como una valoración de uno mismo en comparación a los demás. La autocompasión, en cambio, se fundamenta esencialmente en el reconocimiento de nuestras semejanzas y de nuestra humanidad compartida para, desde esa conciencia, desarrollar una actitud bondadosa, amable y equilibrada que esté disponible siempre hacia uno mismo; y así, desde esa disposición constructiva y amable consigo mismo, extenderla hacia los demás.
La autocompasión es un factor protector para nuestra salud. Por ejemplo, una investigación con estudiantes universitarios encontró una asociación directa e indirecta (a través de la percepción de bienestar y síntomas depresivos) entre el nivel de autocompasión y la conducta suicida (Kelliher et al., 2018). También se han encontrado asociaciones entre mayor autocompasión y menor consumo de alcohol en adultos jóvenes (Saarinen et al., 2019).

El desarrollo de la capacidad de percibir lo que ocurre con el otro y la habilidad de comprender "la mente y la emocionalidad" son fundamentales en el proceso de la compasión (Figura 1). La sola empatía, sin avanzar hacia la compasión, tiene el riesgo de generar malestar empático y burnout, lo que favorece la generación de juicios negativos hacia el otro e inercia actitudinal (Klimecki \& Singer, 2012). La acción compasiva, en cambio, permite la restauración del equilibrio personal. Dicha restauración es dependiente de la existencia de un soporte social, organizacional o comunitario que favorezca el que se pueda actuar prosocialmente.

Es importante igualmente distinguir empatía de compasión. Para propósitos de esta distinción, las neurociencias cognitivas han identificado las diferencias neurobiológicas entre ambas (Preckel et al., 2018). En la compasión se activan, frente al sufrimiento de otra persona, áreas cerebrales relacionadas con la Teoría de la Mente, de la empatía y el área de la recompensa o actitudes prosociales (altruistas). Sabemos que hay una asociación entre patrones conductuales y cerebrales asociados a empatía y compasión. En el caso de la empatía por el dolor, se incrementa la actividad en áreas relacionadas con emociones negativas (percepción y sentir el sufrimiento) como la ínsula anterior y la corteza media cingulada anterior. En la compasión, por otra parte, se incrementa la actividad en áreas de emociones prosociales y positivas (coincidente con querer mejorar la situación de sufrimiento); así, se activa una red estriato ventral, núcleo pálido y corteza orbitofrontal media, áreas que son ricas a su vez en receptores de oxitocina y vasopresina, asociados con el cuidado amoroso (Chierchia \& Singer, 2017).

Estas investigaciones neurobiológicas nos están permitiendo comprender mejor la naturaleza de estas capacidades, y por cierto permiten orientar su entrenamiento. Por ejemplo: investigadores en el área de la meditación han analizado las diferencias entre meditadores expertos en una línea que favorece la amabilidad como es la "Meditación de la Bondad Amorosa" (Loving-Kindness Meditation, LKM), mostrando que aquellos expertos presentan un incremento funcional en áreas de receptividad al sufrimiento, pero también en zonas que favorecen la conducta prosocial. Por el contrario, los novicios tienen una menor activación de ambas zonas y en cambio incrementan el área relacionada con la teoría de la mente. Experimentos muestran también que, al inducir un 
estado compasivo en adultos, las respuestas de estas personas frente a dilemas sociales tienden a ser más altruistas en comparación a grupos controles (Weng et al., 2013).

Estos hallazgos han permitido plantear que la compasión, además de ser una motivación prosocial que favorece el desarrollo de relaciones saludables, funciona como una capacidad de auto-regulación emocional que, a través de la activación de emociones positivas relacionadas con la recompensa y afiliación, permite modular el riesgo de estrés empático (Engen \& Singer, 2015). En otras palabras, la compasión cuida tanto al que la ofrece como a quien la recibe.

\section{¿Cuál es la relevancia de la compasión para la atención en salud centrada en la persona?}

La investigación ha mostrado que las intervenciones basadas en la compasión otorgan beneficios a los pacientes y a los profesionales de la salud, lo cual, además de los beneficios a nivel humano, permitiría también reducir los costos de la atención en salud (Galante et al., 2014).

De este modo, la investigación se ha dirigido hacia dos ámbitos: intervenciones psicoterapéuticas basadas en el desarrollo de la compasión, por un lado, y por el otro a aquellas destinadas al desarrollo de la compasión como habilidad clínica.

La revisión sistemática de Kirby (2017) señala al menos seis intervenciones basadas en la compasión: la Terapia Centrada en la Compasión (Compassion-Focused Therapy [CFT]) (Gilbert, 2014); la Meditación de la Bondad amorosa (Loving-kindness meditation [LKM]) (Fredrickson et al., 2008; Hoffmann et al., 2011); Mindfulness y Autocompasión (Neff \& Germer, 2013); el Entrenamiento en el Cultivo de la Compasión (Compassion Cultivation Training [CCT]) (Jazaieri et al., 2014; Brito-Pons et al., 2018); el Entrenamiento de la Compasión Basado en la Cognición (Cognitively-Based Compassion Training [CBCT]) (Pace et al., 2013); y el Cultivo del Equilibrio Emocional (Kemeny et al., 2012). Todas con investigaciones en curso o terminadas que, en general muestran eficacia.

En el ámbito de la educación de profesionales de la salud se ha enfatizado últimamente la relevancia de la compasión como habilidad clínica. En el caso norteamericano, un estudio evidencia que hasta un $53 \%$ de las personas piensan que los médicos no atienden de manera compasiva (Lown et al., 2011). Al respecto, Sinclair et al., (2016) preguntaron a pacientes sobre qué implicaba un médico compasivo. De acuerdo a lo reportado se indicaron 5 aspectos:

1. Atributos médicos: médico auténtico, amoroso, abierto, honesto, franco, cuidadoso, comprensivo, tolerante, amable, que acepta/acoge.
2. Atributos relacionales: busca entender al paciente, sus necesidades y su persona.

3. Atributos comunicacionales: comunicación no verbal afectuosa, respetuosa, amable, demuestra cuidado por el otro. Comunicación dialógica.

4. Atributos compasivos: preocupación y acciones en pro del confort físico, espiritual, emocional, afectivo durante el tratamiento. Preocupado por el contexto familiar y financiero.

5. Resultados atribuidos a una relación compasiva: alivio del sufrimiento, mejorar bienestar, mejorar autocuidado.

Para los tratantes, la compasión en la actividad clínica se asocia con una mejor adherencia terapéutica, mejor diagnóstico clínico, mayor confianza personal sobre la acción médica y mejora en la satisfacción laboral y permanencia en el trabajo. Krasner et al. (2009) evidencian cómo el entrenamiento en habilidades compasivas en médicos familiares se asocia a reducción de errores médicos y reduce el uso innecesario de recursos. Por otra parte, Orellana-Ríos et al. (2018) muestra que el entrenamiento en atención plena y compasión mejora la satisfacción laboral, el autocuidado y reduce el estrés laboral. Lo mismo señalan Krogh et al. (2019) respecto de la resiliencia en medicina.

Para Lown (2016), el foco de la educación médica de pre y postgrado en este tema debería ser el desarrollo de habilidades y atributos que favorezcan la compasión a lo largo de la carrera y que, de manera transversal, guíen el currículo. Es fundamental adicionar un liderazgo importante en los ambientes de práctica clínica para que favorezcan y refuercen el quehacer compasivo de los estudiantes. De acuerdo con esto, Fernando et al. (2017) realizaron un experimento exploratorio para estimular la compasión en estudiantes. A todos se le midió su disposición compasiva al inicio; al grupo control se les "entrenó" haciendo escuchar por 10 minutos una reflexión sobre la relevancia del servicio al público, mientras que al grupo experimental se les entrenó por 10 minutos con ejercicios de meditación basados en la meditación de bondad amorosa. A ambos grupos se les midió el estado emocional tras el entrenamiento. Luego de cada meditación, se les presentó a ambos grupos viñetas con situaciones de pacientes difíciles para que tomaran una decisión sobre cómo abordarlos. Los resultados manifestaron en el grupo experimental una tendencia mayor a tomar conductas y considerar aspectos de las cinco áreas señaladas en el estudio de Sinclair (2016).

Lo que se busca con este tipo de intervenciones es que los estudiantes adquieran el "hábito compasivo" y que desarrollen la 
intuición, es decir, la capacidad individual de actuar; decidir apropiadamente entre alternativas diversas, sin demandar un proceso consciente y deliberante, y sin necesariamente seguir una norma o regla (Kahneman \& Klein, 2009). Esto es, la intuición que se basa en la compasión para hacer lo correcto incluso en situaciones de incertidumbre y que implica una capacidad perceptiva amplia, la cual se nutre del conocimiento explícito y tácito para mejorar la comprensión del otro y del contexto.

En Chile, respecto a las competencias del perfil del egresado de Medicina, la Asociación de Facultades de Medicina proponen que la compasión sea un rasgo a desarrollar en los profesionales médicos. (Asofamech, 2016).

Con todos estos antecedentes queda en evidencia la necesidad de reflexionar sobre la compasión en la práctica médica, puesto que existe una necesidad de transformar la atención de salud incorporando no sólo cambios estructurales básicos como son infraestructura, equipos, etc.; sino también destacando y reivindicando la importancia de los cambios personales y organizacionales en la dirección de la humanización y la atención centrada en la persona.

La compasión es una capacidad relevante para la atención clínica y ya contamos con evidencias preliminares de ello tanto en intervenciones hacia el paciente como hacia estudiantes. Para poder desarrollar y potenciar esto necesitamos entrenamiento para los profesionales, técnicos y administrativos, y también políticas institucionales y cambios organizacionales que favorezcan las actitudes compasivas. Además, se requiere continuar investigando al respecto, con el propósito de contribuir con conocimiento para una verdadera transformación y humanización de la atención en salud.

\section{Referencias}

ASOFAMECH (2016) Competencias comunes para los egresados de las Escuelas de Medicina ASOFAMECH. Accedido en http://www. carlosjorquera.com/CompetenciasPerfilASOFAMECHfinal.pdf el 08 de julio, 2019.

Bedregal P, Passi A\& Chang M. (2016). The use of complementary therapies in Chile: Results from the National Health Survey 2010-2011. The Journal of Alternative and Complementary Medicine 22, A1-A142.

Brito-Pons G, Campos D. \& Cebolla A. (2018). Implicit or Explicit Compassion? Effects of Compassion Cultivation Training and Comparison with Mindfulness-based Stress Reduction. Mindfulness 9, 1494-1508.

Brito-Pons G. \& Librada-Flores S. (2018). Compassion in palliative care: a review. Current Opinion in Supportive and Palliative Care 12, 472-479.
Chierchia G. \& Singer T. (2017). The neuroscience of compassion and empathy and their link to prosocial motivation and behavior. In Decision Neuroscience, pp 247-257 eds. Dreher J.C. \& Tremblay L. Academic Press

Constand MK, MacDermid JC, Dal Bello-Haas V. \& Law M. (2014). Scoping review of patient-centered care approaches in healthcare. BMC health services research 14, 271-279.

Engen HG. \& SingerT. (2015). Compassion-based emotion regulation up-regulates experienced positive affect and associated neural networks. Social cognitive and affective neuroscience 10, 1291-1301.

Fernando AT, Skinner K. \& Consedine NS. (2017). Increasing compassion in medical decision-making: can a brief mindfulness intervention help? Mindfulness 8, 276-285.

Fredrickson BL, Cohn MA, Coffey KA, Pek J. \& Finkel SM. (2008). Open hearts build lives: positive emotions, induced through loving-kindness meditation, build consequential personal resources. Journal of Personality and Social Psychology 95, 1045-62.

Galante J, Galante I, Bekkers MJ \& Gallacher J. (2014). Effect of kindness-based meditation on health and well-being: a systematic review and meta-analysis. Journal of consulting and clinical psychology 82, 1101-14.

Gilbert P. (2014). The origins and nature of compassion focused therapy. British Journal of Clinical Psychology 53, 6-41.

Gilbert P. (2015). Mindful compassion: how the science of compassion can help you understand your emotions, live in the present, and connect deeply with others. London: Robinson.

Gilbert P, Catarino F, Duarte C, Matos M, Kolts R, Stubbs, J, Ceresatto L, Duarte J, Pinto-Gouveia J. \& Basran J. (2017). The development of compassionate engagement and action scales for self and others. Journal of Compassionate Health Care 4, 4.

Gómez-Batiste X, Mateu S, Serra-Jofre S, Molas M, Mir-Roca S, Amblàs J, Costa X, Lasmarías C, Serrarols M, Solà-Serrabou A, Calle C. \& Kellehear A. (2018). Compassionate communities: design and preliminary results of the experience of Vic (Barcelona, Spain) caring city. Annals of palliative medicine 7, S32-S41.

Hangartner D. (2015). Neuroscience of Compassion: Challenges and Opportunities. Dharma Drum Journal of Buddhist Studies 16, 123-140.

Hill TE. (2010). How clinicians make (or avoid) moral judgments of patients: implications of the evidence for relationships and research. Philosophy, Ethics, and Humanities in Medicine 5, 11. 
Hoffmann SG, Grossman P \& Hinton DE. (2011). Loving-kindness and compassion meditation: Potential for psychological intervention. Clinical Psychology Review 31, 1126-1132.

Institute of Medicine (US) Committee on Quality of Health Care in America. (2001). Crossing the Quality Chasm: A New Health System for the 21 st Century. Washington, D.C: National Academy Press.

Jazaieri H, McGonigal K, Jinpa T, Doty JR, Gross JJ. \& Goldin PR. (2014). A randomized controlled trial of compassion cultivation training: Effects on mindfulness, affect, and emotion regulation. Motivation and Emotion 38, 23-35.

Jinpa T. (2015). A fearless heart: how the courage to be compassionate can transform our lives. New York: Avery Publishing Group.

Juengst E, McGowan ML, Fishman JR. \& Settersten Jr RA. (2016). From "personalized" to "precision" medicine: the ethical and social implications of rhetorical reform in genomic medicine. Hastings Center Report 46, 21-33.

Kahneman D. \& Klein G. (2009). Conditions for intuitive expertise: a failure to disagree. American psychologist 64, 515-526.

Kelliher Rabon J, Sirois FM. \& Hirsch JK. (2018). Self-compassion and suicidal behavior in college students: Serial indirect effects via depression and wellness behaviors. Journal of American College Health 66, 114-122.

Kemeny ME, Foltz C, Cavanagh JF, Cullen M, Giese-Davis J, Jennings P, Rosenberg E, Gillath O, Shaver P, Wallace BA. \& Ekman P. (2012). Contemplative/emotion training reduces negative emotional behavior and promotes prosocial responses. Emotion 12, 338-250.

Kirby JN. (2017). Compassion interventions: The programmes, the evidence, and implications for research and practice. Psychology and Psychotherapy: Theory, Research and Practice 90, 432-455.

Klimecki O. \& SingerT. (2012). Empathic distress fatigue rather than compassion fatigue? Integrating findings from empathy research in psychology and social neuroscience. In Pathological altruism, eds. Oakley B, Knafo A, Mahavan G. \& Wilson D.S. pp. 368-383. New York, NY, US: Oxford University Press.

Krasner MS, Epstein RM, Beckman H, Suchman AL, Chapman B, Mooney CJ. \& Quill TE. (2009). Association of an educational program in mindful communication with burnout, empathy, and attitudes among primary care physicians. Jama 302, 1284-1293.

Krogh E, Medeiros, S, Bitran, M. \& Langer Ál. (2019). Mindfulness y la relación clínica: pasos hacia una resiliencia en medicina. Revista médica de Chile 147, 618-62
Kuhn M, Letunic I, Jensen LJ. \& Bork P. (2015). The SIDER database of drugs and side effects. Nucleic acids research 44, D1075-D1079.

Lown BA, Rosen J. \& Marttila J. (2011). An agenda for improving compassionate care: a survey shows about half of patients say such care is missing. Health Affairs 30, 1772-1778.

Mladovsky P, Srivastava D, Cylus J, Karanikolos M, Evetovits T, Thomson S. \& McKee M. (2012). Health policy responses to the financial crisis in Europe: impact and implications for policy. World Health Organization.

Neff KD. \& Germer CK. (2013). A pilot study and randomized controlled trial of the mindful self-compassion program. Journal of clinical psychology 69, 28-44.

Neff K. D. \& Germer C. (2017). Self-Compassion and Psychological Wellbeing. In Oxford Handbook of Compassion Science, ed. Doty J. Oxford University Press.

Orellana-Rios C., Radbruch L., Kern M., Regel Y., Anton A., Sinclair S. \& Schmidt S. (2018). Mindfulness and compassion-oriented practices at work reduce distress and enhance self-care of palliative care teams: a mixed-method evaluation of an "on the job"program. $B M C$ palliative care 17,3 .

Pace TW, Negi LT, Dodson-Lavelle B, Ozawa-de Silva B, Reddy SD, Cole SP, Danase A, Craighead L. \& Raison C. L. (2013). Engagement with cognitively-based compassion training is associated with reduced salivary C-reactive protein from before to after training in foster care program adolescents. Psychoneuroendocrinology 38, 294-299.

Ricard M. (2016). Altruism: the power of compassion to change yourself and the world. Main: Atlantic Books.

Saarinen A, Keltikangas-Järvinen L, Cloninger CR, Veijola J, Elovainio M, Lehtimäki T, Raitakari O. \& Hintsanen M. (2019). The relationship of dispositional compassion for others with depressive symptoms over a 15-year prospective follow-up. Journal of affective disorders 250, 354-362.

Sinclair S, Norris JM, McConnell SJ, Chochinov HM, HackT F, Hagen N A, McClement S. \& Bouchal SR. (2016). Compassion: a scoping review of the healthcare literature. $B M C$ palliative care 15, 6 .

Thompson I, Amatea E \& Thompson E. (2014). Personal and contextual predictors of mental health counselors' compassion fatigue and burnout. Journal of Mental Health Counseling 36, 58-77.

Weng HY, Fox AS., Shackman AJ, Stodola DE, Caldwell JZ, Olson MC, Rogers GM. \& Davidson RJ. (2013). Compassion training alters altruism and neural responses to suffering. Psychological science 24, 1171-1180. 